\title{
A New Technique for Jaw Thrust in Unconscious Craniofacial Trauma Patients
}

\author{
Gaurav Verma M.D.S. \\ Senior Lecturer, Department of Oral and Maxillofacial Surgery, Himachal Institute of Dental Sciences, \\ Paonta Sahib, Himachal Pradesh, India. \\ *Corresponding Author E-mail: gauravjournals107@gmail.com
}

\begin{abstract}
The loss of consciousness is one of the commonly encountered emergency situations in maxillofacial trauma patients. Apart from benign syncope, there are other causes for unconsciousness. Majority of these patients have intact respiratory muscle activity, but the airway patency is compromised due to airway obstruction by tongue fall back. Traditionally head tilt-chin lift and jaw thrust techniques were employed to maintain patency of the airway. Head tiltchin lift technique involves tilting the head backward and thus is dangerous in case of suspected cervical spine injury in trauma cases. Jaw thrust is an efficient method and can be performed in cervical spine injury cases. But it requires continuous forward pull on mandible till the time patient regains consciousness; which is physically strenuous for rescuer. The purpose of this article is to present a new technique for jaw thrust which is based on similar principle of traditional jaw thrust. The advantage is that, jaw thrust can be maintained for an extended time period in these patients.
\end{abstract}

Keywords: Head Tilt-Chin Lift; Jaw Thrust; Bag-Valve Mask Ventilation; Endotracheal Intubation; Unconsciousness.

\section{Introduction}

Unconsciousness is defined as lack of response to sensory stimulation. The loss of consciousness leads to depression of vital signs of the patient, depressed protective reflexes and inability to maintain patent airway. Irrespective of the cause of unconsciousness, the management protocol for these patients starts with P-Position, A-Airway, B-Breathing, and CCirculation and D-Definitive management [1].

Head tilt-chin lift and jaw thrust techniques were described in the literature to maintain the patency of the airway. These maneuvers are based on the principle to stretch the tissue between the larynx and mandible, elevate the base of the tongue away from the posterior pharyngeal wall. These techniques relieve anatomic airway obstruction caused by soft tissues in $80 \%$ of the unconscious patients. Head tilt-chin lift technique involves tilting the head backward and thus is dangerous in case of suspected cervical spine injury. Jaw thrust is efficient method and can be performed in cervical spine injury cases. [1] But, it requires continuous forward pull on mandible till the time patient regains consciousness; which is physically strenuous for rescuer. The purpose of this article is to present a new technique for jaw thrust which is based on similar principle of traditional jaw thrust.

\section{Technique}

After establishing unconsciousness and proper positioning of the patient, oral cavity should be cleared of excessive saliva, blood, vomitus and debris to clear airway. An assistant maintain the mandible in open and protruded position. Topical local anesthetic is applied at selective sites of wire placement (mentioned below). 26-gauze sterilized prestretched stainless steel wires; $15 \mathrm{~cm}$ in length are passed around maxillary canines and mandibular 2nd premolars. For each tooth individual wire is passed from buccal side to palatal/ lingual side from distal contact (Fig. 1, Step 1) and then passes it back to buccal side from mesial contact (Fig. 1, Step 2). The two ends of the wires are twisted together (Fig. 2, Step 3). Similar wires were placed on four teeth (as mentioned above). 


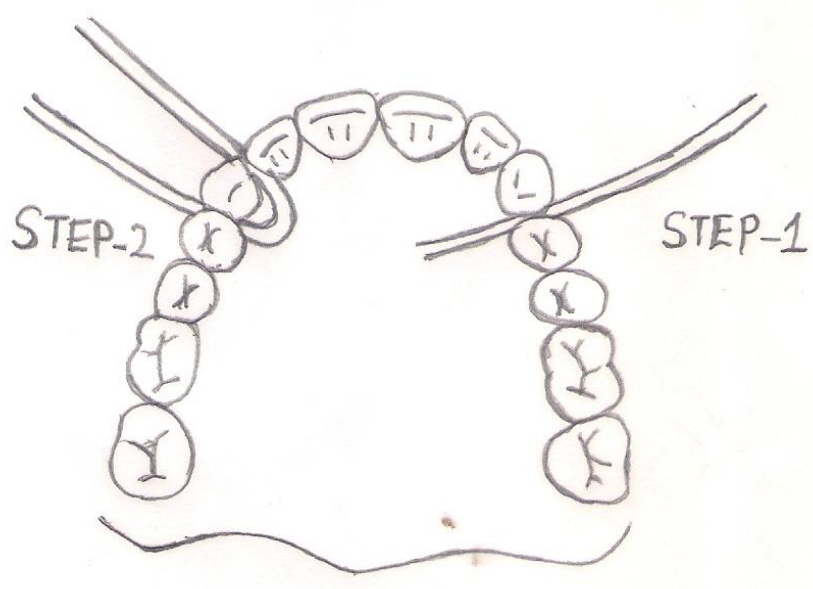

Fig. 1: Step 1: Wire passed from buccal to palatal/lingual side through distal contact. Step 2: Wire passed back to buccal side through mesial contact.

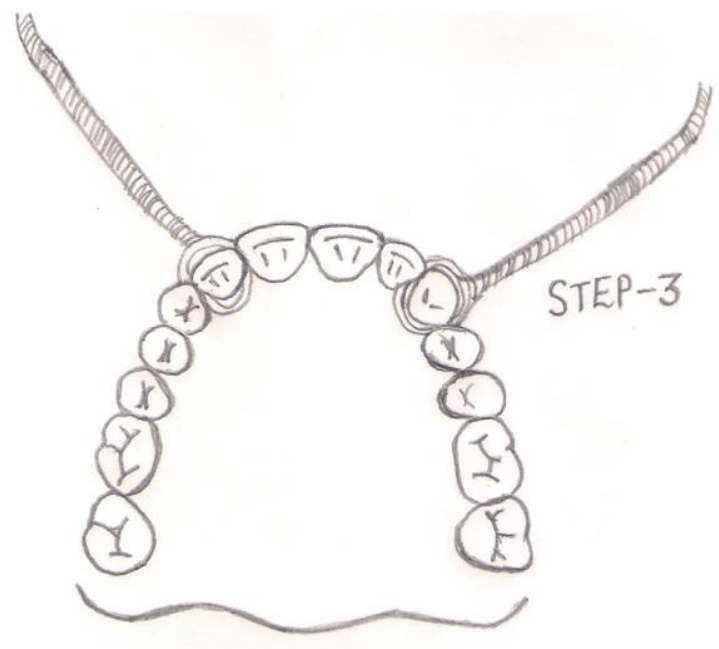

Fig. 2: Step 3: Two ends of the wire twisted together.

After the placement of individual wires, the mandible is protruded $(9-10 \mathrm{~mm})$ forward and these wires are twisted together to achieve maxillo-mandibular fixation and excess wires are cut short. This is similar to Gilmer's direct wiring method for maxillo-mandibular fixation (MMF) used in maxillo-facial fractures. In Gilmer's method the aim is to achieve pre-morbid occlusion (Fig. 3), but here the aim is to do maxillo-mandibular fixation (MMF) in protruded mandibular position irrespective of occlusion (Fig. 4, Step 4).

In the absence of suitable teeth (as described above), other teeth can be used in maxilla and mandible. The wires on maxillary teeth should be placed $9-10 \mathrm{~mm}$ anterior to selected mandibular teeth $(9-10 \mathrm{~mm}$ is approximate value of protrusive movement of mandible). The basic principle is that, this allow maxillo-mandibular fixation (MMF) with mandible in protruded position.

To further enhance the air exchange, plastic cheek retractor can be used to retract the lips and cheek. Oral cavity should be constantly cleared by placing suction through the buccal vestibule. After placement of wires the patient should be closely monitored to check the efficacy of measures taken for A-B-C-D. Once the patient is stable, these wires can be removed easily. 


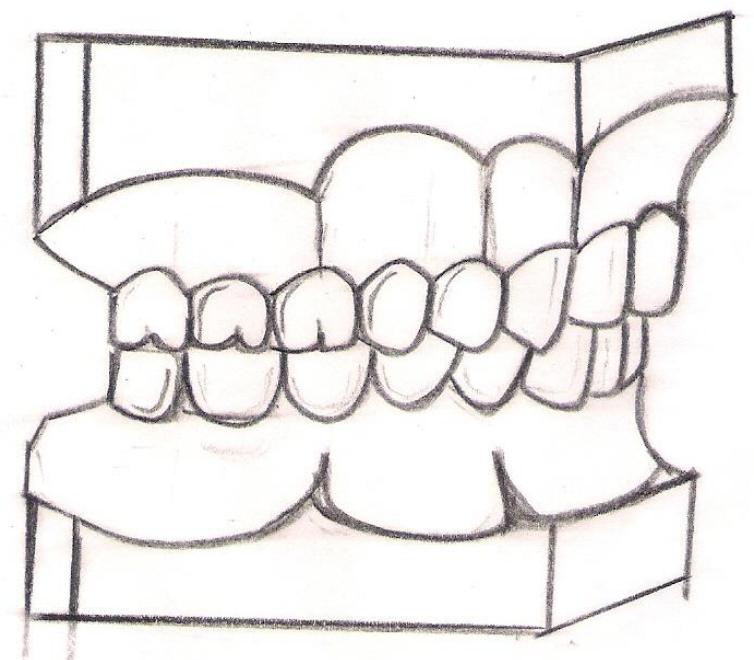

Fig.3: Maxillo-mandibular relationship in normal occlusion.

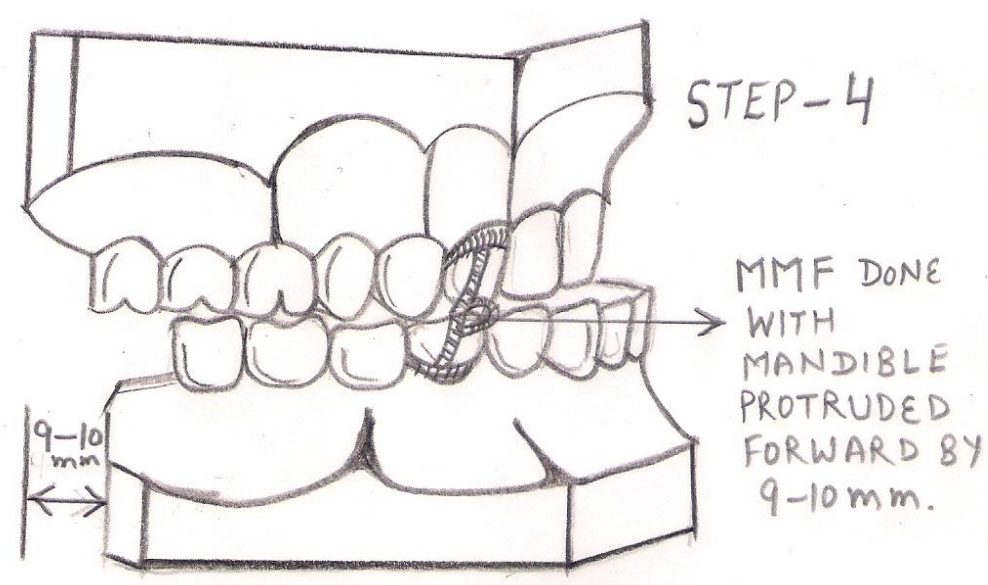

Fig. 4: Maxillo-mandibular fixation with mandible in protruded position.

\section{Discussion}

Once a clinician identifies airway compromise in a patient, definite measures should be taken to maintain the patency of the airway. Initially this is accomplished by use of airway maneuvers, suctioning the airway, and using airway adjunct $[2]$.

Chin lift, head tilt-chin lift and jaw thrust techniques are the airway maneuvers to maintain the patency of the airway [1, 2]. Research conducted during past 20 years, established that conventional head tilt-chin lift and jaw thrust provides the most consistently reliable airway [1].

Suctioning will remove the debris, secretions etc to clear the airway. Airway adjuncts include use of oral and nasopharyngeal airways. Both these airways are designed to displace the tongue and soft tissues forward so that the patient can breathe through or around them. The oropharyngeal airway has the disadvantage that a semiconscious patient may not be able to tolerate it. Also, it can induce vomiting in patients with intact gag reflex. This should be determined beforehand by stimulating the soft palate and posterior tongue gently and observing for the presence of gag response [2].

The nasopharyngeal airway is especially useful in patients who cannot maintain the patent airway and have intact gag reflex. However, there is risk of passage of nasopharyngeal airway intracranially through a base of skull fracture. 
Although, the risk of intracranial placement is less, major craniofacial trauma may be considered as a relative contraindication for use of nasopharyngeal airway [2].

Bag valve mask ventilation is used in patients who are evidently hypoxic or not breathing spontaneously (apnoeic) despite the use of airway maneuvers, suction and airway adjuncts. Bag valve ventilation is required in these apnoeic patients to pre-oxygenate them prior to placement of endotracheal intubation [2].

From the review of literature it is clear that, an unconscious patient may be breathing spontaneously, inadequately or may not be breathing at all. In unconscious patient the cause for airway obstruction is tongue fall back, as the patient is not able to maintain it in forward position. So, A-airway and B-breathing, in patients who are having efficient respiratory muscle activity can be maintained by maintaining the patency of airway.

Similar to conventional jaw thrust this new technique ensures the mandible in protruded position to allow for constant forward pull on the tongue and maintain the patency of the airway. These means to maintain airway patency are based on spontaneous respiration by patient. In the absence of spontaneous respiration, oxygenation should be performed with artificial means. Although one may argue against the need for wiring in an emergency situation at the expense of AAirway. But it must be noted that this technique in itself ensure airway patency. Firstly, suctioning the oral cavity to remove blood clot, saliva, vomitus and other debris clear the airway. Secondly, placement of the wires require mouth opening, and during this procedure an assistant help the operator by holding the mandible in opened and protruded position i.e. jaw thrust is incorporated constantly. Thirdly, bag-valve mask ventilation can be performed with the wiring in place, if the need arises. This technique will take 4-5 minutes for an operator because it require placement of 4 wires only. If need arises, the two twisted wires can be cut in quick time (10-15 seconds).

It must be noted that, this method should exclude cardio-pulmonary arrest, for which the definitive management include cardio-pulmonary resuscitation (CPR). Also, this method should not be employed in craniofacial trauma patients who are actively vomiting and bleeding heavily intraorally and in patients with benign vasovagal syncope.

\section{Conclusion}

In conclusion, it can be stated that, this new technique is based on the principle of jaw thrust. The clinical efficacy of jaw thrust is scientifically proven. This new technique can be used to maintain patent airway in unconscious craniofacial trauma patients for extended time period.

\section{References}

[1] S. F. Malamed, Medical Emergencies in the Dental Office, Mosby, Reed Elsevier India 2008.

[2] R. J. Fonseca, R. V. Walker, N. J. Betts, H. D. Barber and M. P. Powers, Oral and Maxillofacial Trauma, Elsevier Saunders, St. Louis, Missouri, 2005. 